\title{
Pressure Rise Control in Axial Ducted Fan by Acoustic Noise Modeling
}

\author{
T.V. Rama Murthy ${ }^{(1)}$, Y.G. Srinivasa ${ }^{(2)}$, M. Govardhan ${ }^{(2)}$, G.R. Srinivasa ${ }^{(3)}$ \\ (1) Aerospace Electronics Division, National Aerospace Laboratories, Bangalore 560 017, India \\ (2) Department of Mechanical Engineering, Indian Institute of Technology, Chennai 600 036, India \\ (3) Department of Mechanical Engineering, BMS College of Engineering, Bangalore 560 019, India
}

\begin{abstract}
A control strategy for maintaining the pressure rise within certain limits in an axial ducted fan has been described. Experiments reveal that the spectrum of the acoustic noise contains predominantly the peak frequency of the broad band rotor noise. The variation of the amplitude of the envelope of a bandpass filter output tuned to the peak frequency with changes in throttle position and impeller speed has been studied. An off-line Least Squares Estimation algorithm fits a discrete time linear model for predicting the amplitude of the envelope. An error signal between the predicted amplitude of the model and its expected value for maximum pressure rise activates the throttle valve servo. Gain Scheduling of the model parameters in terms of the throttle position and the impeller speed is implemented for wider operating range of the machine.
\end{abstract}

\section{NOMENCLATURE}

$f_{p t} \quad$ - peak frequency of the broad band rotor noise spectrum

$N \quad$ - impeller speed in RPM

$p \quad$ - pressure ratio - outlet pressure/inlet pressure

$\Delta p \quad$ - outlet pressure - inlet pressure

$p_{m}$ - maximum value of $p$ for a given $N$

$P_{i}, Q_{i}$ - discrete time model parameters $(i=1,2)$

$S_{p t} \quad$ - amplitude of the envelope of bandpass filter output for full traverse of throttle (also referred to as envelope signal $S_{n x}$ )

$S_{x n} \quad$ - normalized envelope given by $\frac{\left(S_{p t}\right) \text { at any given value } x, N}{\left(S_{p t}\right) \text { at } x=35 \mathrm{~mm}, N=2750 \mathrm{RPM}}$

$S_{P \text { MAX }}$ - value of $S_{x n}$ for a given $\left(N, p_{m}\right)$

$x(t) \quad$ - low pass filtered throttle position sensor signal, mm

$y(t)=S_{n x}$

$x_{n} \quad$ - sample value of $x(t)$

$y_{n} \quad$ - sample value of envelope signal

$\hat{y}_{n} \quad$ - estimate of $y_{n}$

$\hat{S}_{n x} \quad$ - estimate of $S_{n x}$

\section{INTRODUCTION}

The area of active stabilization in axial flow machines has been attracting the attention of many researchers in the fields of gas turbine engines, off-shore plants, power plants, tunnel ventilation and clean rooms. Greitzer ${ }^{1}$ proposed a mathematical model of an axial flow compressor explaining the phenomena of stall and surge. According to Epstein, ${ }^{2}$ active stabilization of compressors basically consists of introducing a feedback controller in order to increase the system damping, so that machines operate in a previously forbidden and unstable region of the compressor map. This new approach has been adopted on a series of laboratory models by Ffowcs Williams, ${ }^{3,4}$ Dugundji, ${ }^{5}$ Pinsley, ${ }^{6}$ Paduano $^{7}$ and Rama Murthy ${ }^{8}$. Some of these techniques are highly complicated and their implementation is expensive. Further, these techniques are targeted towards aircraft applications. Acoustic noise generated in compressors and fans has been studied with emphasis on active noise control by Warnaka, Koopmann, ${ }^{10}$ Byron $^{11}$ and others. The emphasis in these works is on noise control and not on performance optimization of the machine.

Some static empirical formulae are available in reference 12 relating volume flow and static pressure to sound power level for fans. The modeling takes into account the dynamics involved in the physical process and enables faster response to be introduced into the control action. The operation of the fans/compressors becomes unstable due to changes in flow rate, inlet pressure, molecular weight of the gas and changes in the impeller speed. The authors were mainly motivated to use the acoustic properties of the axial ducted fan because the acoustic noise reflects the trend of these influencing parameters. Therefore, modeling of the acoustic noise will be a useful technique in the area of fans and compressors. The control of pressure rise by acoustic noise modeling also results in simpler methods based on the complexity-benefit considerations and instability avoidance concepts. $^{13}$

\section{EXPERIMENTAL SET-UP}

The schematic of the axial ducted fan set-up is shown in Fig. 1. The axial ducted fan set-up consists of a bell mouth orifice, a duct, a fan, an induction motor, a flat-type throttle valve and static pressure taps. The flat type throttle valve is coupled to a DC permanent magnet (DC/PM) motor shaft with a cam arrangement (Fig. 2) to allow the throttle valve to have a linear motion along the axis of the duct. The motor shaft is geared to a servo potentiometer for sensing the throttle position. A feedback speed servo system has been built around the DC/PM motor. The DC/PM motor starts rotating on giving a speed reference signal resulting in a linear motion 